the elemental echo would certainly be less than 60 spot diameters, rather than the 1890 which Milwright suggests.

The use of pulse shaping would, of course, improve matters considerably in this respect, but would possibly introduce the second problem to which Milwright refers. The signal from one storage tube could be processed so that synthetic pulses of fixed length and amplitude, produced in response to signals above a selected amplitude, would be applied to the input of the second storage tube. In these circumstances, any such signal, once stored, would be retained until it passed off the target by virtue of the motion of the observing ship. Interference might thus be stored and could confuse the picture. If the original signal were used to build up the true track, then by setting the loop gain of the storage cycle to be less than unity, a trace which would diminish in intensity with time would be observed. By varying the loop gain, the trace persistence could be adjusted so that interference would be prevented from building up excessively.

As I have already said in my conclusion to the article, such a system may add confusion rather than clarity to a complex situation, but this can best be determined by experiment. The method would at least have the advantage of being additive to the normal relative display. In a situation where it is not contributing useful information it could be switched out to leave the relative display.

\title{
Sundials for Navigators
}

\author{
from Captain Mario Bini \\ (Italian Navy)
}

I. Having some time ago been requested to design a sundial for the Hydrographic Institute of Genoa (Fig. I) I went through various books and found that the methods indicated for drawing sundials were all based on graphical constructions. Although quite interesting these methods have the inconvenience of all graphical solutions, that they require constructions which, besides being complicated, turn out to be rather inaccurate, especially when lines cross at small angles, as inevitably happens outside a limited central area of the drawing. To avoid this I worked out a numerical system, related to the familiar methods of astro-navigation, which, by means of simple formulae, gives the coordinates of the points necessary to design a sundial. As the bibliography I examined is, of course, limited, I am not in a position to state whether this method of calculation is new or not; I describe it here hoping it may interest people who have sometimes wondered how sundials are made.

2. Sundials are based on the principle that a gnomon stylus casts its shadow, over a datum plane, along a line having a direction depending only on time, provided the stylus is set parallel to the Earth's axis.

The stylus OS (Fig. 2) is contained in the plane of all hour circles, its shadow corresponding to different positions of the Sun (such as I, 2, 3) along the same 


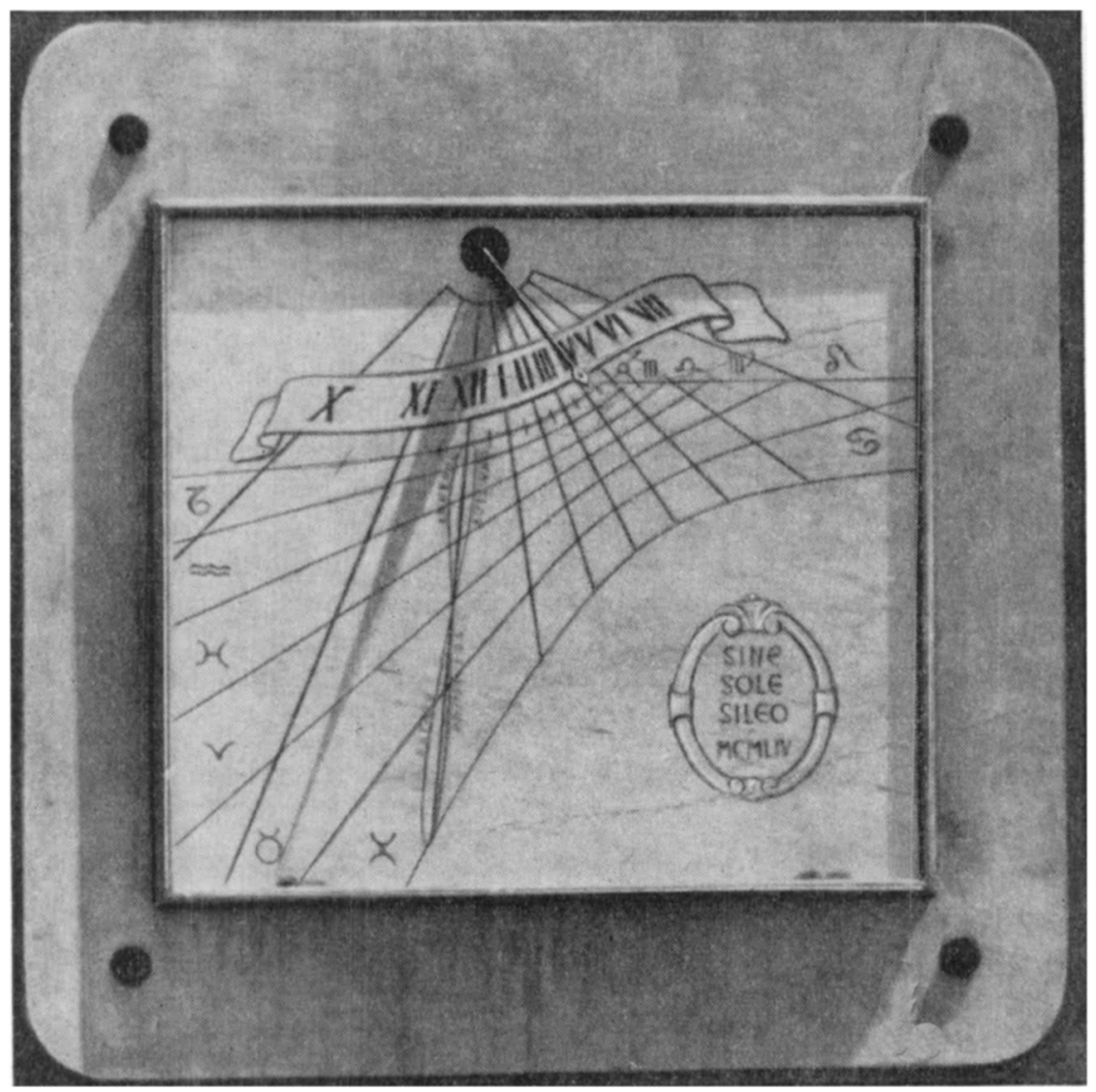

FIG. I. The sundial at Genoa

hour circle, and therefore at the same true local time, always falls on the same line $\mathrm{AA}^{\prime}$, which is in fact the intersection of the hour circle considered with the plane of the sundial. The changes in the Sun's declination make the length of the

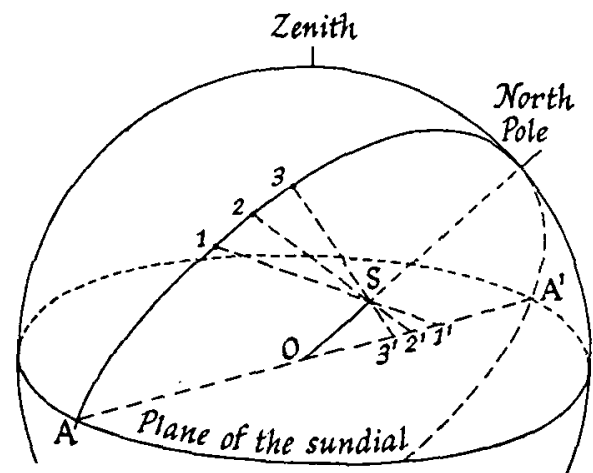

FIG. 2. The stylus and shadows of the Sun shadows o1', o2', 03' vary, but do not affect its direction.

The sundial therefore consists of a plane, generally horizontal or vertical, from which the stylus originates; from the basis of the stylus radiates a set of lines, one for each hour. These are the 'hour lines' (Fig. 3).

Connecting all the points of the hour lines on which the top of the stylus casts its shadow on given days of the year, another series of curves can be obtained which are called 'declination lines' and represents the 
path described by the shadow of the stylus top on those given days. They are all conic sections; the one corresponding to declination $0^{\circ}$ is a straight line (intersection of the plane of the celestial equator with the plane of the sundial).

The hour and declination lines form the typical pattern of sundials, which is completed by a curve having the shape of a flattened figure 8 , which is a graphical representation of the equation of time; it enables one to keep account of the difference between true and mean time.

3. Let us first consider a horizontal sundial (Fig. 4): OS is the stylus, which being parallel to the Earth's axis forms, with the plane, an angle equal to latitude $\varphi .1$ being the stylus length and $C$ the foot of the vertical through $\mathrm{S}$, we have $\mathrm{OC}=1 \cos \varphi$, and $\mathrm{CS}=1 \sin \varphi$.

The direction $O C$, being the vertical projection of the stylus (parallel to the Earth's axis), represents true North. In a datum moment the shadow of $S$ will fall on a point $T$, at a distance from $\mathrm{C} C \mathrm{CT}=\mathrm{SC}$ cot

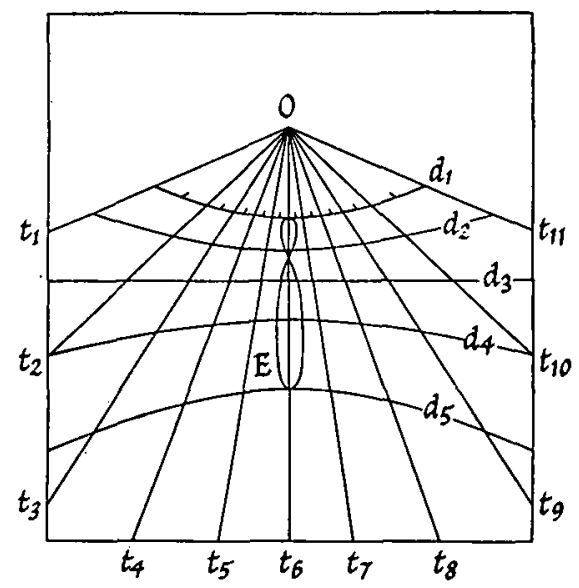

$t_{1}-t_{11}$ Hour lines

$d_{1}-d_{5}$ Declination lines

E Curve of the equation of time

0 Stylus origin

FIG. 3. Hour lines

$h=l \sin \varphi \cot h$ and in a direction CT given by Sun's az $\pm 180^{\circ}$ where $h$ is the altitude and $\mathrm{Z}$ the azimuth of the Sun.

If therefore, taking $\mathrm{C}$ as centre we were to:

(a) draw a series of circles having radii equal to $1 \sin \varphi \cot h$

(b) draw in a pencil of lines

(c) label each circle with the corresponding altitude

(d) label each line with $Z \pm 180^{\circ}$,

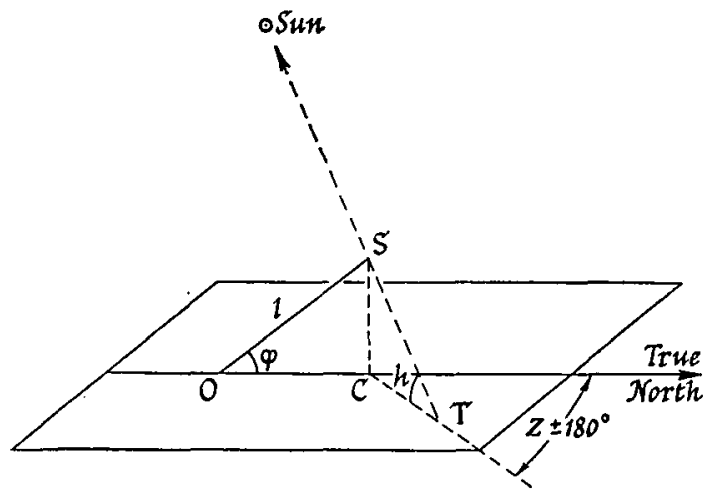

FIG. 4. A horizontal sundial

we should obtain a pattern (Fig. 5) by means of which, knowing the altitude and azimuth of the Sun, the position of the shadow of the stylus top $S$ could be directly marked. 
In order to make the method completely numerical the $\mathrm{OC}$ direction can be taken as $x$ axis, the perpendicular through $C$ as $y$ axis (Fig. 5); so we have $x=\mathrm{CT} \cos Z=1 \sin \varphi \cot h \cos Z$ and $y=\mathrm{CT} \sin Z=1 \sin \varphi \cot h \sin Z$, and putting $1 \sin \varphi=K_{1}$ :

$$
\begin{aligned}
& x=K_{1} \cot h \cos Z \\
& v=K_{1} \cot h \sin Z
\end{aligned}
$$

Orientating the axis as in Fig. 5 the stylus origin $\circ$ has coordinates $x=-1 \cos \varphi$, $v=0$; for the signs of $x$ and $y$ it can be noted that:

$x$ is positive if $Z$ is between $90^{\circ}$ and $270^{\circ}$ passing through south, and negative passing through north (all this refers to northern latitudes; in the southern hemisphere the necessary alterations have to be made).

$y$ is positive if $Z>180^{\circ}$, negative if $Z<180^{\circ}$.

4. As pointed out in para 2, two sets of lines-hour and declination lineshave to be drawn on the sundial; the two things can be done together simply by calculating the Sun's altitude and azimuth for 'round' hours of chosen days of the year, as for example:

(a) $\begin{cases}2 \text { I } & \text { March } \\ 23 & \text { September }\end{cases}$

(b) 21 June

When Sun's declination is zero

(c) 21 December

When Sun's declination is $23^{\circ} 27^{\prime}$ North

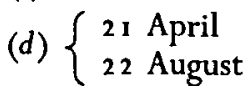

When Sun's declination is $23^{\circ} 27^{\prime}$ South

(e) $\begin{cases}24 & \text { October } \\ 18 & \text { February }\end{cases}$

When Sun's declination is $\frac{23^{\circ} 27^{\prime}}{2}$ North

When Sun's declination is $\frac{23^{\circ} 27^{\prime}}{2}$ South

If more declination lines are desired other values can be chosen in place of $(d)$ and $(e)$ so as to divide in a greater number of equal parts the $0^{\circ} \div 23^{\circ} 27^{\prime}$ intervals of declination.

In order to read the time more accurately it may be convenient to increase the

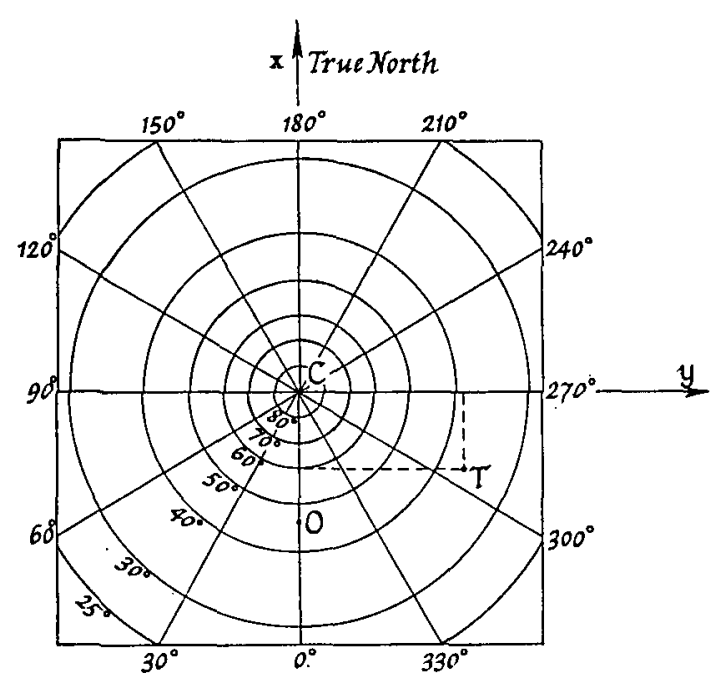

FIG. 5 hour pattern; to do this without drawing too many lines it is sufficient to mark only a small part of an hour line at suitable intervals (every I 5 or 20 minutes, for instance) on the declination line nearest to the stylus (Fig. 3), as this line is cut by the shadow of the stylus on any day of the year.

The position of these intermediate hour marks could of course be obtained using the formulae; more simply they can be determined by plotting the distances amongst the successive round hours (measured 
along the declination line nearest to the stylus) against time. From this plot the distances at which the subdivisions fall can be read off with sufficient accuracy.

5. Proceeding as above the sundial would give true local time, which differs from the zone time Z.T. by an amount equal to the sum of the equation of time and the time zone correction T.Z.C. : as this sum can be as much as three quarters of an hour, and in any place varies during the year within a range of half an hour, it is evidently convenient to eliminate these two causes of difference and so have Z.T. directly from the sundial.

This can be easily done for T.Z.C. (which being constant can be directly accounted for while calculating the local hour angle corresponding to 'round' Z.T. hours), but is not possible for the equation of time, which varies continuously during the year; the only possible way is that of considering it equal to zero and then providing the sundial with a device to keep account of this variable quantity that has been disregarded.

The device is a graphical representation of the equation of time obtained by drawing the locus of points on which the shadow of the end stylus top falls at $12^{h}$ Z.T. on the various days of the year. To draw this curve the azimuth and altitude of the Sun must be calculated for Z.T. $12^{\text {h }}$ of the first day of each month, the corresponding positions of the shadow calculated and put down, and finally a smooth curve drawn across them (Fig. 6). It is convenient to write nearby the corresponding seasons.

When reading the time indicated by the sundial one must follow along the

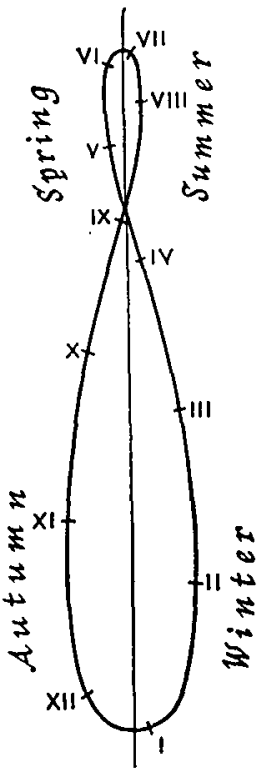

Curve of the equation of time for a horizontal sundial in lat. $45^{\circ}$ North ( 1 = 1st. January, $11=1$ st. February, etc.)

FIG. 6. Curve of the equation of time declination line, on which lies the shadow of the stylus top, until one meets the curve; knowing from the date which side of the curve to use, one can see from it the approximate correction* to be applied mentally to the time read on the sundial in order to have the Z.T.

6. So far we have dealt with horizontal sundials; in case of vertical sundials a similar approach can be used, except that the pattern of azimuths and altitudes is formed respectively by:

Vertical lines, originated by the intersection of vertical planes, passing through the stylus end $S$, with the plane of the sundial.

Conic sections, originated by the intersection of the projection through $S$ of parallels of equal altitude with the plane of the sundial.

* Keeping in mind that the shadow will be or was over the curve at $12^{\mathrm{h}} \mathrm{Z}$.T. it is easy to see whether the correction has to be added or subtracted from the time indicated by the shadow. 
Owing to this fact it would not be so easy to draw the pattern of altitudes and azimuths as it is for horizontal sundials; the problem is further complicated by the fact that there is one more element to be accounted for, the orientation of the plane of the sundial, unless it is exactly east-west. As vertical sundials are always fixed to walls, one must normally keep account of the orientation, as walls are never-except exceptionally-lying due east-west. The orientation of the wall has to be measured unless it can be obtained with the necessary accuracy from a large-scale map, possibly taking it from the orientation of the streets (this of course if the walls are parallel and perpendicular to the street).

If no suitable map can be used for this purpose the orientation has to be measured with some instrument, and this can be done in many ways. A simple one is probably that of choosing a suitable position along the wall extension and measuring the angle between the direction of the Sun and that of the wall (with a tachometer or a theodolite in any moment, with a sextant only if the Sun can be observed at sunrise or sunset, in order not to introduce tilt errors); if no

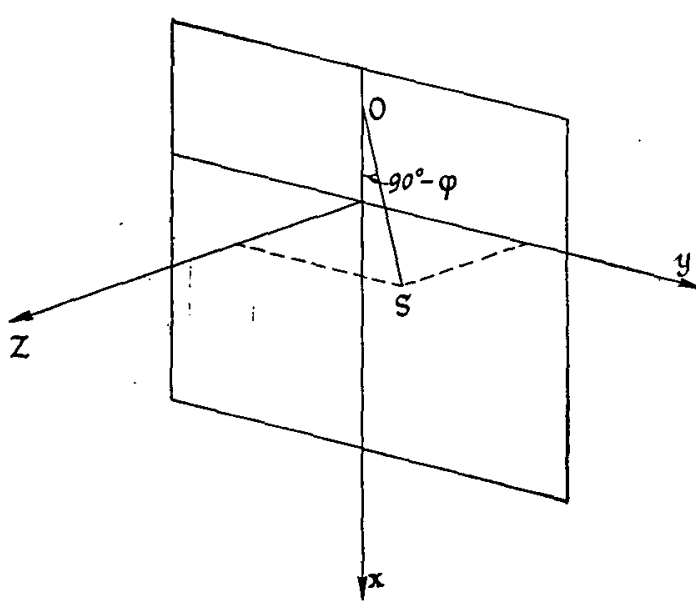

FIG. 7. Vertical sundial diagram instruments are available, observing the time at which the shadow of a plumb-line falls on a line, parallel to the wall, drawn over an horizontal plane (the plumbline must be in the vertical plane passing through the horizontal line).

In both cases the exact time must be noted and then, using the familiar methods of astro-navigation, the azimuth of the Sun calculated and the orientation of the wall obtained : several measurements are necessary to avoid blunders.

7. For vertical sundials it is convenient to put the axis as in Fig. 7:

$x$ is the vertical axis, passing through the stylus origin o

$y$ is the intersection of an horizontal plane through $S$ with the plane of the sundial

$z$ is perpendicular to the sundial plane

As azimuth of the sundial plane we will consider that of $z$ axis, indicating it by $\alpha$ : the azimuth to be entered in the calculations is therefore that of the perpendicular to the wall, away from it.

The formulae giving the position of the shadow of $\mathrm{S}$ are in this system (omitting their demonstration for the sake of brevity):

where

$$
\begin{aligned}
& x=k_{2} \sec \beta \tan h \\
& y=k_{3} \sec \beta \sin Z
\end{aligned}
$$

$$
\begin{aligned}
k_{2} & =1 \cos \varphi \cos \alpha \\
k_{3} & =1 \cos \varphi \\
\beta & =|\alpha-Z|
\end{aligned}
$$


The sign of $x$ is always positive (the $y$ axis is in fact the line on which the shadow falls when the Sun's altitude is $0^{\circ}$ ); as for the sign of $y$ it must be noted that OS being parallel to the Earth's axis and, therefore, contained in the plane of the meridian, the shadow of the stylus will be cast over the $x$ axis when the Sun's azimuth is $180^{\circ}$; so $y$ will be positive when $Z>180^{\circ}$ and negative when $Z<180^{\circ}$.

8. Once the final drawing is made, the actual building of the sundial has to be done. The sundial can be engraved in marble or-especially if it is a small horizontal one-on a bronze or brass plate (well polished and then covered with several coats of good marine varnish it will keep shining for years), or in any other suitable material taking in due consideration the quality of craftmanship available. It can also be simply painted on a wall or on tiles.

It is traditional to design on sundials the zodiacal signs, a latin motto such as 'sine sole sileo' or 'nihil sine sole nihil sine umbra' or 'horas non numero nisi serenas', \&c.

And so we come to the last point, that of correctly setting the sundial up.

For horizontal sundials it is necessary that:

The sundial is exactly horizontal.

The sundial is rotated-always keeping it exactly level-in such a way that at Z.T. I $2^{\text {hoo }}$ the shadow of the stylus end falls exactly on the curve of the equation of time corresponding to the date.

The stylus has a length equal to $l$ and has its origin in the point $x=-1 \cos \varphi$, $y=0$.

The stylus forms an angle with the plane of the sundial equal to latitude and is contained in the vertical plane passing through the $180^{\circ}$ azimuth line.

Vertical sundials have only to be fixed to the wall, keeping them straight; for them the problem is limited to the correct positioning of the stylus, and this operation has of course to be done before securing the sundial to the wall.

It should be noted that the stylus never lies in a plane perpendicular to the sundial except the very rare case of a wall exactly oriented eastwest. The following data have to be used for the correct positioning of the stylus (Fig. 8):

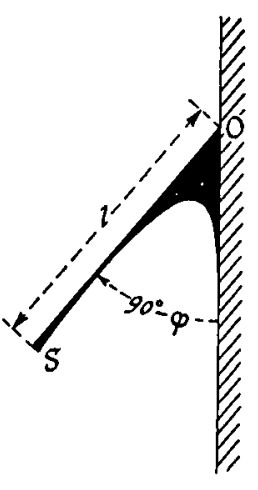

View along y axis

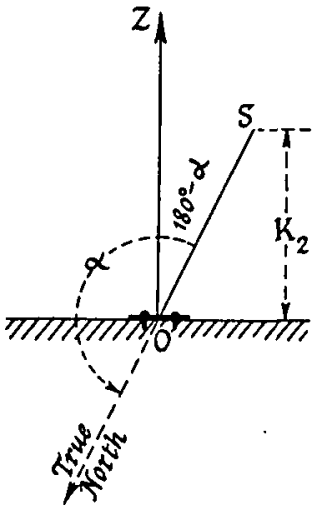

View along $\mathrm{x}$ axis

FIG. 8. Positioning of the stylus for a vertical sundial

The origin must be in the point $x=-1 \sin \varphi, y=0$.

Its length must be equal to 1 .

The angle CÔS must be $90^{\circ}-\varphi$.

The angle SĈZ must be $180^{\circ}-\alpha$ if $\alpha<180^{\circ}$ or $\alpha-180^{\circ}$ if $\alpha>180^{\circ}$.

The distance from $\mathrm{S}$ to the plane of the sundial must be equal to $k_{2}$

$(l \cos \varphi \cos \alpha)$. 
9. It is worthwhile pointing out that a common feature to all sundials is that once calculated they can be enlarged or reduced by multiplying or dividing all the coordinates, and the stylus length, by the same number; this can be done even more simply by choosing a suitable scale for the drawing.

In view of this and also in order to save time the stylus length $l$ can be omitted in the calculations (i.e. considered equal to $\mathrm{r}$ ): once the calculations are done an approximate draft of the sundial can be made and having decided its definite size the final drawing can be carried out adopting for the axis the scale that will give the desired size.

\section{Dr. R. d'E. Atkinson, of the Royal Greenwich Observatory, comments:}

This paper may stimulate a number of readers to take up a rather fascinating hobby, but it seems worth while to point out that there are in fact a good many possibilities in addition to those actually indicated. It is not as widely known as it should be that any given sundial can always be moved to a different latitude and longitude from those for which it was designed, and can still be set up so as to work correctly.

If two identical dials are computed and made for any given place, and one of them is set up correctly there, the other can be taken to any other place on the Earth, and provided it is set up parallel to the first one in every way, it will always record the same apparent time as that one at the same absolute instant. The two must of course be truly parallel, and the line which is horizontal and north-south on the one will not in general be (locally) north-south or (locally) horizontal on the other. But if they really are parallel, the Sun's rays will always hit them both in just the same way at any given instant, and the shadow of the gnomon will fall on the plate in just the same way in both cases. It may of course be found that the horizon gets in the way at some times, and the theorem also ignores the effect of the solar parallax; but sundials regularly do ignore this, and indeed ignore the much larger refraction also. Apart from these disturbing factors, a parallel displacement of a few thousand miles is entirely without significance; we are all of us displaced from the centre of the Earth, in one direction or another, by nearly 4000 miles.

We may note, incidentally, that all sundials everywhere already have their slanting gnomon-edges parallel, since they are all parallel to the Earth's axis. Two of the three rotational degrees of freedom are thus determined anyway, and the third is necessarily a rotation about that line. The third angle, which may (for example) be defined by specifying the tilt of any E.-W. line on the plate, may be readily computed by methods similar to those of Captain Bini's paper.

The theorem we have stated has a considerable variety of application in detail. For example, if we have a sundial which is correctly computed and set up at some station, and if we want it to read the standard time for the zone (after applying the Equation of Time, of course) instead of the local time, we need only rotate it about the slanting gnomon-edge, through an angle equal to our longitude difference from the standard meridian; it will then be truly parallel to an identical sundial correctly set up on that meridian and will record what that one would. Quite generally in fact, if any correctly-computed sundial is rotated about its gnomon-edge through any angle $\theta$ (degrees), its readings at any given instant will be changed by $\theta / 15$ hours, whatever the actual time or date may be. Thus if we construct it with suitable bearings to give this kind of rotation, and if we limit 
the rotation to two suitable positions $15^{\circ}$ apart, we can set it over to the east, in spring, so that it will read Summer Time, and can set it back again in the autumn, at a touch. It is really just like setting a watch. Alternatively, we can provide the rotation with a scale and pointer, and set it day by day (or week by week) so that the dial readings are always corrected for the Equation of Time. The scale can, if we wish, be marked out in terms of the dates when the Equation reaches certain values.

It is clear that the angle between the plate and the gnomon can be chosen freely, at any latitude. At any given station, one particular angle will result in a horizontal dial, and its complement will result in a vertical dial for a south wall; but one can tilt the plate in any other way with respect to the gnomon too, so long as one computes the positions of the hour-lines correspondingly. On can in fact (and put in this way it is really obvious) not only compute a dial for a vertical wall in any azimuth but also for a non-vertical wall or bank, again in any azimuth; and when the instrument has been made to this specification one can transport it anywhere else and set it up parallel to the attitude for which it was designed; it will always record the same time as it would have at its place of origin. One can then rotate it about its gnomon, and it will record the time for any other longitude instead; for example, the time for the place to which one actually has brought it, or the time of the standard meridian for that place, or whatever one wishes. Thus one can take a sundial designed and made as an ordinary vertical one for a south wall in Edinburgh, and can transport it to Capetown and set it up as an ordinary horizontal one there; if it recorded local time at Edinburgh, a parallel set-up at Capetown would leave it still recording Edinburgh time, but rotating it (about the slanting gnomon-line, as always), through the longitudedifference of about $21^{\circ}$, will make it read local time for Capetown, and incidentally will in this case make the plate horizontal as well. The change of latitude from $+56^{\circ}$ to $-34^{\circ}$ just converts 'vertical' into 'horizontal', and vice versa; but one could have stopped anywhere on the way too and set it up as an 'inclined' sundial, and it would still have worked. It is true that when the plate is not horizontal the Sun may sometimes be 'below' or 'behind' it although it is above the horizon; but this is regularly accepted with wall sundials anyway. If one can mount it clear of such obstacles as walls, and if one carries the gnomon on through the plate and graduates the latter on both sides, it will read correctly even when the Sun is 'below' the plate; but not even then, unfortunately, when it is below the horizon.

Clearly, a sundial is something that could be mass-produced, with only three or four models to cover the whole Earth. This, however, would be a most regrettable thing to do; the real charm of a sundial is its individuality. The opposite application of our basic theorem is much better; if one has room in one's garden, one can set up a number of sundials, each computed as an ordinary horizontal one for some selected place on the globe and erected parallel to its orientation in that place, and each will then show that place's local time. Whenever the Sun changes from one side of the plate to the other it will be sunrise or sunset at the place in question, whatever the time of year, and in general one will thus need to graduate both sides. In England, the Auckland dial will have its 'lower' side pointing nearly 'upwards', and will record almost exclusively New Zealand night hours; but it will record them. And the different tilts of the various plates will demonstrate the Earth's roundness in a way that should be rather attractive, particularly to navigators. 